\title{
A Regulatory-Based Approach to Safety Analysis of Unmanned Aircraft Systems
}

\author{
James T. Luxhøj and Ahmet Öztekin \\ Department of Industrial and Systems Engineering \\ Rutgers University, Piscataway, NJ USA \\ jluxhojarci.rutgers.edu, aoztekinarci.rutgers.edu
}

\begin{abstract}
Unmanned Aircraft Systems (UAS), the new frontier in civil aviation, add another dimension to the ever-increasing complexity of the current National Airspace System (NAS) in the United States. The future inclusion of private and commercial operations of the UAS into the NAS, unavoidably, raises safety concerns. As the NAS becomes increasingly more complex and constrained, the associated hazard and safety risk modeling must also mature in sophistication. Thus, there is a need for advanced studies focusing on risk-based system safety analysis of emergent UAS operations. This paper presents a regulatory-based integrated approach to system safety and risk analysis of the UAS operations and their interaction with the current NAS and the future Next Generation (NextGen) Airspace.
\end{abstract}

\section{Introduction}

The National Airspace System (NAS) in the United States is increasingly becoming a complex array of commercial and general aviation aircraft, unmanned aerial systems, reusable launch vehicles, rotorcraft, airports, air traffic control, weather services, and maintenance operations, among others. This increased system complexity necessitates the application of systematic safety risk analysis methods to understand and eliminate where possible, reduce, and/or mitigate risk factors.

As the NAS becomes increasingly more complex and constrained, the associated hazard and safety risk modeling must also mature in sophistication. Thus, there is a need for advanced studies focusing on risk-based system safety analysis of emergent Unmanned Aircraft Systems (UAS) operations. This paper presents a regulatorybased integrated approach to system safety and risk analysis of the UAS operations and their interaction with the current NAS and the future Next Generation (NextGen) Airspace. Four distinct yet closely related areas of analysis comprise the main thrust of the proposed approach: taxonomy development, causal factor identification, database development, and modeling complex uncertainty.

Safe integration of UASs into the NAS presents significant challenges to all parties of the aviation community. Although the main thrust of this immerging technology originates from entrepreneurs, both civilian and military, the burden of the safe integration arguably lies on the shoulders of regulatory agencies such as Federal Aviation Authority (FAA). The question of safety associated with this integration arises 
principally due to the unknowns of potential hazards and associated risks while operating in the NAS and interacting with existing NAS users. Formally, UAS is defined as: A device used or intended to be used for flight in the air that has no onboard pilot, which is a clarification of the existing Aircraft definition, 14 CFR §1.1, which indicates that UAS operations are governed by the existing regulations.

In the past, when incidents or accidents occurred, a forensic approach was undertaken in the hazard analysis phase of the system safety approach. Heinrich suggested the "domino" theory of accidents [2]. This view changes the focus of accident investigations toward the events involved, rather than the conditions surrounding the accident environment. The objective is for analysts and investigators to understand the accident phenomenon on the basis of the chain of events that had occurred. The National Transportation Safety Board (NTSB) uses a variant of the sequence of events approach in their analysis of aircraft accidents.

While such a forensic approach has merit and has been important and useful to system safety analysts in the past, it is very scenario driven and quite dependent on the contextual factors involved in the specific incident or accident. As such, the safety recommendations emerging from such a forensic analysis may be quite aircraft type or airport specific.

The system safety approach involves an identify-analyze-control method of safety as opposed to a "fly-fix-fly" approach [1]. One key hypothesis of this paper is that the system safety approach is better suited to safety analysis of new classes of aircraft where data is sparse and operations are limited. In particular, UAS represent a new class of aircraft that is emerging in the current NAS and that will most likely be an integral component of the U.S. Next Generation (NextGen) Air Transport System and the Single European Sky (SESAR). The basic building block for both NextGen and SESAR involves creating an operating environment in four dimensions: latitude, longitude, altitude, and time [3]. It is envisioned that the NextGen will facilitate the move to "free-flight" where satellite-derived positioning data is transmitted via a digital data link to the ground and every aircraft in the sky will then select its own flight path for optimal speed, fuel consumption and turbulence avoidance [3].

\section{System Safety Methodology}

A second hypothesis of this paper is that safety hazards may be derived top-down as opposed to bottom-up. Rather than collecting hazard information from a case-by-case or scenario approach, especially for novel aircraft systems where such data is usually not available, the conjecture is that the Title 14 Code of Federal Regulations or CFRs may be used to derive hazards as well as their underlying causal factors by utilizing a systems analysis approach.

Thus, this paper proposes a regulatory-based integrated approach to system safety and risk analysis of the UAS operations and their interaction with the current NAS and the future NextGen Airspace. The next sections describe the elements of the proposed approach in more detail. 


\subsection{Development of a System-Level Taxonomy for Categorization of UAS Hazards:}

One of the first steps in the proposed UAS system safety analysis is hazard identification and analysis. To that end, a new hazard taxonomy was developed. This taxonomy, termed the Hazard Analysis and Classification System (HCAS) identifies four main hazard system sources: Airmen, UAS, Operations, and Environment. The basic framework of the proposed taxonomy closes is based on the FAA regulatory perspective (i.e., Title 14, Code of Federal Regulations (14 CFR) chapters on Aircraft, Airmen, Certification/Airworthiness, Flight Operations, etc.). Such an approach uniquely distinguishes the HCAS taxonomy from all other UAS hazard analyses being performed by the Department of Defense (DoD), the RTCA-Special Committee (SC) 203 [4,5], etc.

Safety analysis has a fundamental role to play in the identification of hazard source potentials, the understanding of the underlying causal factors, the likelihood assessment of these factors, the severity evaluation of the potential consequence(s) of mishaps, and the prioritization of mitigations.

A sound system-level safety analysis relies heavily on properly identifying the key components of the area of interest. In particular, the identification of potential hazard sources and sub-sources within the systemic structure of the problem domain should be considered as a fundamentally important step in system safety analysis. Furthermore, since semantics play a crucial role while defining the domain variables, a systematic taxonomy that balances fidelity and generalization provides a solid foundation for a meaningful and relevant system safety analysis. Within this context, we present the HCAS taxonomy specifically designed and developed to identify and categorize individual system-level hazard sources for UAS operations.

HCAS categorizes the UAS hazards consistent with the 14 CFR Sub-chapters, thereby establishing the taxonomy on the FAA regulatory framework. The advantage of the proposed approach is to allow direct association of hazards identified with regulatory requirements or vice versa. Once the system is established, it will not only provide the FAA as well as the UAS community the tools to determine safety and regulatory implications of UAS operating in the NAS, but also fall in directly under the FAA Safety Management System (SMS) Doctrine. Particularly, as described in [6], the taxonomy was uniquely developed but was inspired by the research of Hayhurst et al. [7] and the RTCA Special Committee 203 [5]. 208 hypothesized UAS mishap scenarios provided by the FAA are employed to verify and test the robustness of the taxonomy. These hypothesized scenarios were not detailed or specific operational scenarios but were rather more akin to "thought experiments" of possible UAS mishaps due to their inclusion in the NAS. de Jong et al. [8] present an approach to pushing the boundary between imaginable and unimaginable hazards that keeps the performance of the hazard identification process separate from the hazard analysis and hazard mitigation processes, so the idea of developing "UAS scenario themes" is consistent with the de Jong method for hazard identification. These hypothesized UAS scenarios supported "analytic generalization" and were primarily used to develop concise terminology of system and sub-system hazard sources. The semantics of the hazards were aligned in a general way with the wording of the main CFR chapters and also vetted with industry subject matter experts. 
UAS Hazard Classification and Analysis System (HCAS) - version 3.5

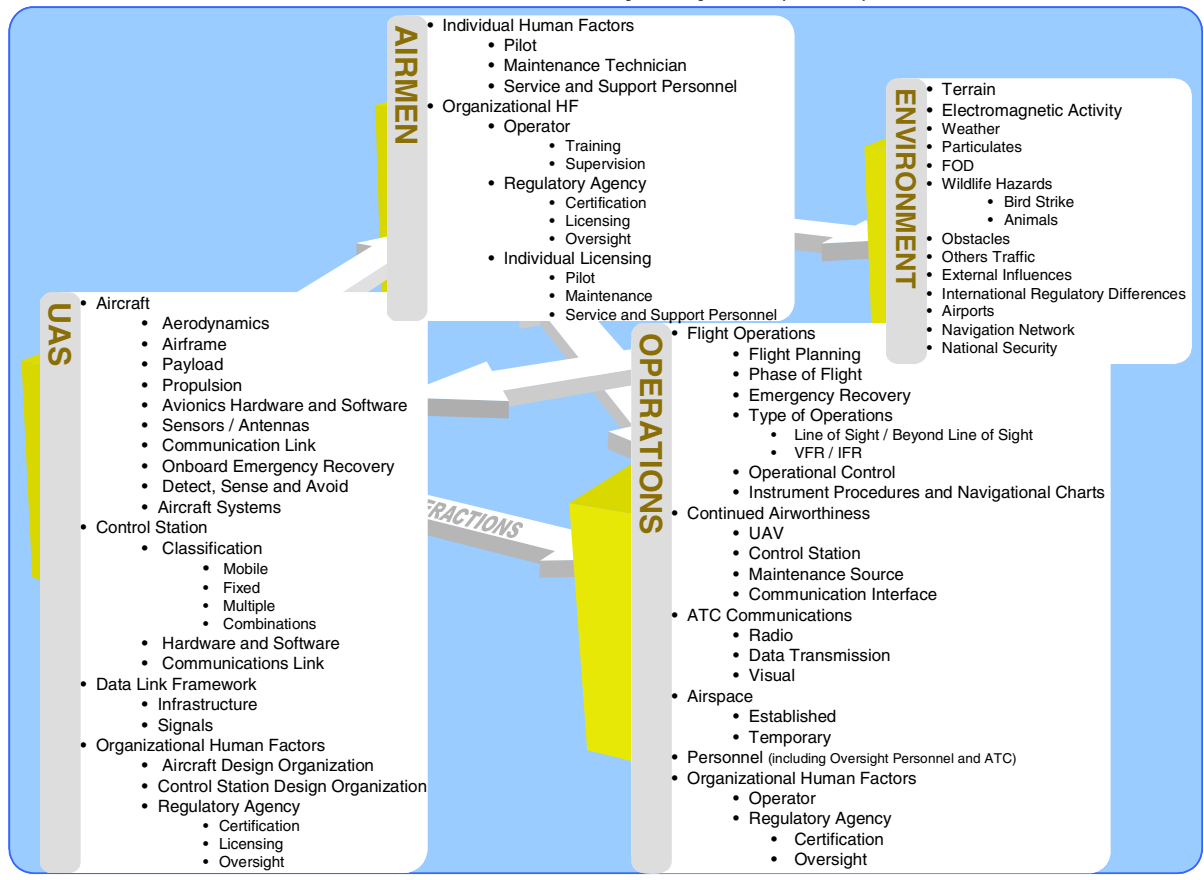

Fig. 1. The current version of the HCAS taxonomy

Using the definition of a hazard as adapted from Leveson [9] to be that a hazard is a state or set of conditions of a system that, together with other conditions in the environment of the system, may lead to an accident (loss event), the HCAS taxonomy was created.

In the current version of the HCAS depicted in Figure 1, four primary hazard sources are identified as UAS, Airmen, Operations, and Environment. For each of the system hazard source potentials, subsystem elements are also identified. For example, for the system hazard source of "UAS", the subsystem hazard sources groups of aircraft, control station, data link, and organizational human factors are included. The notion of a "hazard source" is consistent with "hazardous element" of Ericson's "hazard triangle" and also recognizes that a hazard needs a trigger or initiator to move it from a dormant to an active state, thus focusing on the hazard's "potential" to do harm. Summary papers on HCAS versions 1, 2 and 3 are presented in Öztekin, Luxhøj, and Allocco [6] and in Öztekin, Luxhoj [10].

These influence diagrams may then be used to study the interactions among various causal factors associated with the hazards. Conceptually, HCAS represents a hierarchical structure for UAS hazard sources. In particular, at the very top, there are system-level hazard sources, which, in lower levels, are decomposed into their subsystem-level hazard sources. Since civil UAS operations are relatively new and emergent, databases of mishaps are not readily available. 
The proposed taxonomy depicted in Figure 1 may also be used to construct influence/causal factor diagrams representing hypothetical or notional UAS outcome scenarios. The use of modifiers placed on the HCAS taxonomy elements, such as "inappropriate", "inadequate", etc. may be used to create such an influence diagram.

\subsection{Identifying the Causal Factors - A Regulatory-Based Approach}

Hazards are not causal factors. The decomposition of hazards into their constituent causal factors is another important step in the development of a comprehensive scheme for UAS safety risk modeling. Underlying causes of the hazards, such as failure modes, operator and software errors, design flaws, etc., need to be identified in order to eventually determine the mishap risk and the hazard mitigations.

However, HCAS is not a taxonomy of causal factors. Although the resulting taxonomy for the UAS hazard sources is intended to be generic and inclusive, it represents an inductive reasoning approach with particular emphasis on a given set of UAS hazard scenarios. Hence, to determine a taxonomy of UAS causal factors, which are, strictly speaking, hierarchically at a lower level than hazard sources, we chose to employ deductive reasoning and based our analysis on the current FAA regulations for commercial civil aviation. Knowledge elicitation sessions with subject matter experts are heavily utilized throughout this process. Subsequently, individual causal factors are mapped to the taxonomy of UAS hazard sources resulting in a seamless analysis that is generic enough to cover most possible UAS operational scenarios yet provides the necessary level of fidelity to map their prominent features into a database.

At the crux of our regulatory-based approach lie the following assumptions:

- UAS integration will impact the entire NAS because of the wide-ranges of UAS size, weight, performance characteristics, airspace access, and unique operation issues;

- There are no sufficient data and proven methods to perform UAS safety analysis with the traditional event-driven approach; The regulations provide the essential safety net for the NAS safety;

- There exist a set of causal factors, which can be identified, associated with each relevant regulatory section;

- With proper descriptions of causal factors, the inter-dependencies (linkages) among themselves can be demonstrated;

- These linkages form the basis to assess UAS safety risk analyses that are performed by applying to the current event-driven approach through the Hazard Classification and Analysis System (HCAS) model.

Conceptually, our proposed approach to identify causal factors based on existing regulatory structure represents a hierarchical framework. At the very top, covering the whole NAS, Federal Aviation Regulations (FARs) provide the minimum requirement for safe operations. Within the context of FAR Subchapters, functional models provide fidelity to conceptualize the risk associated with the proposed UAS operations. Consequently, groups of causal factors are identified to outline the underpinnings of each UAS related risk. However, unlike conventional hierarchical methodologies such as Fault Trees, the proposed framework, illustrated in Figure 2, 


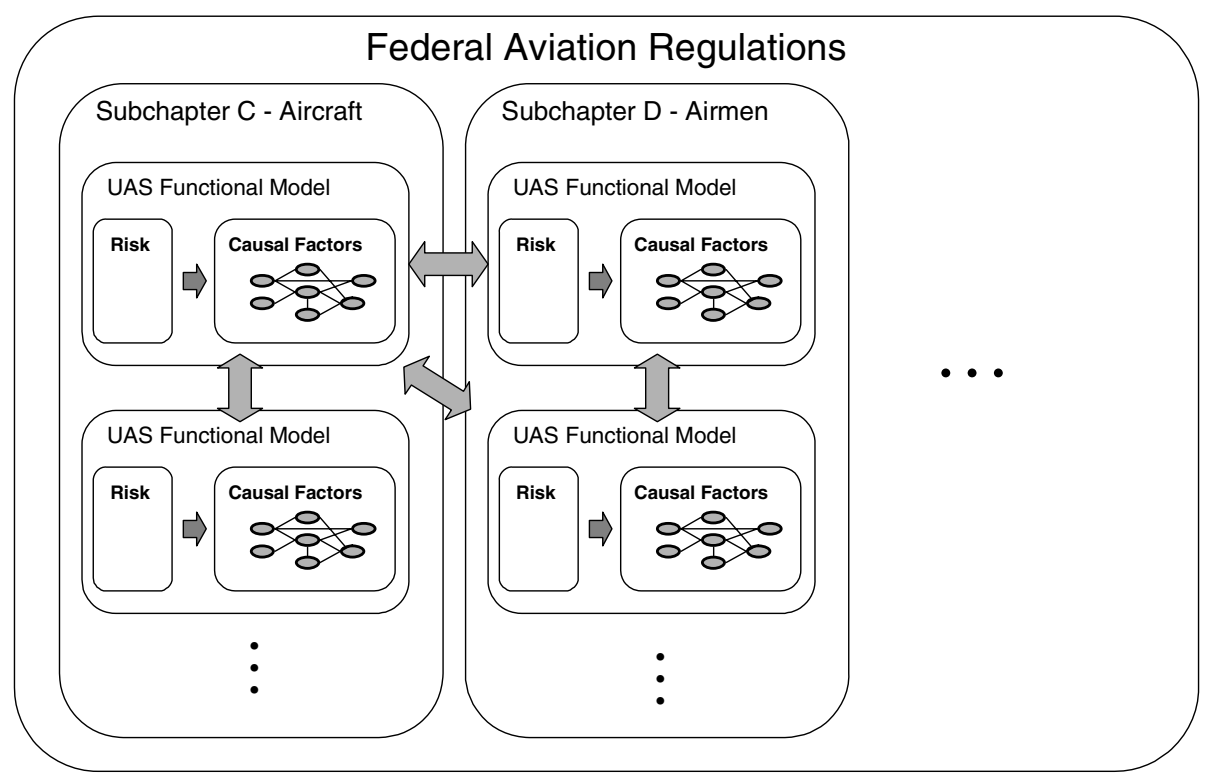

Fig. 2. Hierarchy and interactions within the proposed regulatory-based framework

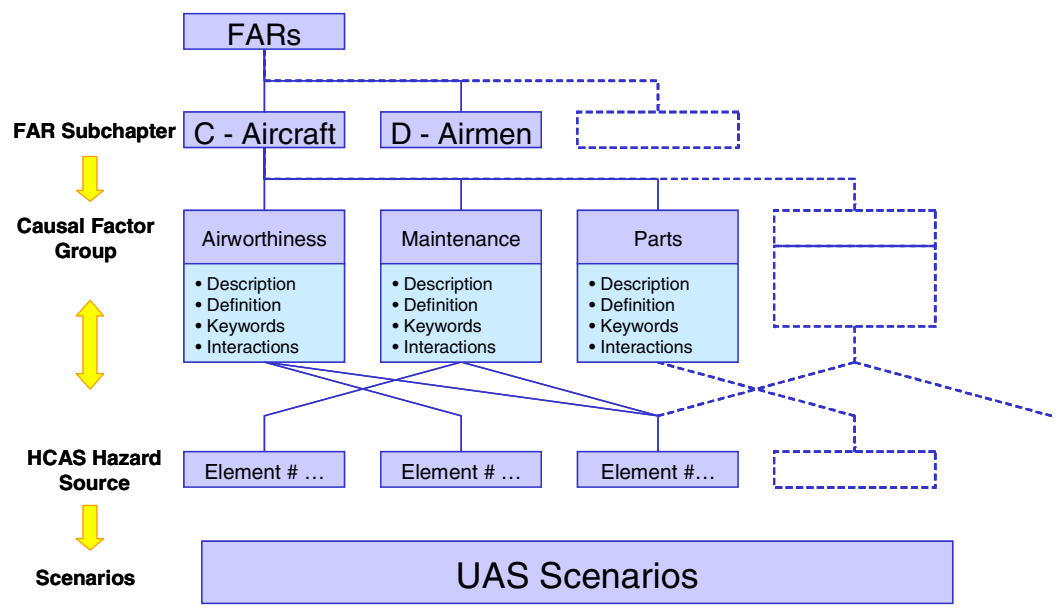

Fig. 3. Causal Factors are the link between regulations and HCAS taxonomy

also emphasizes the interactions and connectivity among various components and compartments comprising the whole domain.

We introduce the notion of a "causal factor cohort" or "grouping" to maintain a system-level approach while structuring the UAS causal factor taxonomy and to establish a viable linkage between HCAS and individual causal factors. The "grouping" emanates from the Title 14 CFR Subchapters. In some cases, it may be that a cohort title coincides with the exact title of a subchapter, such as "Airspace" or "Airmen". In 
other cases, it may be that the title of a cohort may not coincide with the title of a subchapter and be an "analytic construct" that is the result of a creative dialogue by the UAS safety team. In the latter case, such cohort terms as "Design" or "Documentation" may emerge. Since the development of cohort terminology will be a creative process by the UAS safety team, the cohort titles will need some vetting within the aviation community. For each causal factor cohort or grouping, the causal factors within that grouping will be identified, described, defined, and keywords provided. Any interactions with other causal factor cohorts or groups will also be identified. Figure 3 is a notional diagram depicting the causal factor cohort or grouping strategy.

\subsection{UAS Safety Database}

The development of the database for UAS causal factors constitutes a key component of the proposed integrated approach and focuses on identifying keywords associated with individual causal factors. Since the whole research effort has a regulatory skew the proposed analysis is aimed to define some initial boundary conditions for understanding the safety requirements of an emerging technology with possible hazardous impact on current aviation operations. Within the context of a UAS scenario, which could be an accident/incident or an operational application, a collection of possible causal factors can be considered as the feature set defining that particular scenario. For the purposes of text/data mining, keywords will be used to map individual UAS scenarios to representative sets of causal factors. These sets of causal factors could also be employed by a regulatory agency to identify possible areas of concern for safety associated with a specific application for UAS operations. The development of the proposed safety database is currently in its early stages.

\subsection{Causal Probabilistic Modeling of Complex Systems}

Another significant challenge in modern aviation system safety practice is the analytical modeling of emergent operations in the NAS that include the use of a new generation (NextGen) of advanced aircraft and supporting systems, such as very light jets, reusable launch vehicles, unmanned aircraft systems, among others. Since these operations are new, accident and incident data are extremely rare, and alternative modeling approaches to conventional fault tree logic are required to understand the impact of the introduction of these operations into the NAS. Many real-world complex systems are naturally represented by hybrid models, which contain both discrete and continuous variables. The UAS operations within the NAS represent such a complexity. However, current methods for quantifying complex uncertainty demonstrate topological and algorithmic limitations when addressing the interactions of these variables.

Bayesian Networks (BNs) are tools to model uncertainty in the form of a probability distribution imposed by a directed acyclic graph representing the domain of interest. Hence, BNs only address the uncertainty in the form of randomness about a problem domain. However, uncertainty in a typical real-world application has three dimensions: vagueness, ambiguity, and randomness [11] and BNs, being solidly anchored to probability theory, only address one of these dimensions, namely randomness. For instance, 
consider that there is ambiguity regarding the observed evidence associated with some variable in a given Bayesian Network. Such an ambiguity can be modeled by Fuzzy Sets. More specifically, Fuzzy Set theory, introduced by Zadeh in the late sixties [12], proposes a framework to deal with a poorly defined concept in a coherent and structured way. Examples of poorly defined concepts suitable for the application of Fuzzy logic are semantic variables, such as heavy workload, inadequate training, fast, slow, tall, short, etc. Within the context of our current research, Fuzzy Sets present two important features worthwhile for further exploration:

- Fuzzy Sets provide a complete set of tools to partition continuous domains into overlapping membership regions, which result in a much more realistic discretization of the continuous domain in question.

- Uncertainty regarding any empirical observation can be represented as a Fuzzy measure.

Many real-world complex systems include discrete and continuous variables, which poses as an additional challenge while representing the uncertainty associated with the system as an analytical model. Hybrid Bayesian Networks (HBNs), which include both continuous and discrete variables, are a generalization on discrete only Bayesian Networks. HBNs are inherently more suitable for modeling complex systems; such as visual target tracking as in "see and avoid" type of applications where the variables defining location of the target and its speed are inherently continuous and speech recognition where the bits and pieces of processed audio signals are often continuous.

However, HBNs as the generalization of discrete BNs have their own shortcomings that arise when we would like to perform exact inferencing on them. Exact inferencing on general HBNs imposes restrictions on the network structure of the HBN. The state of the art exact inferencing algorithm for HBNs, the Lauritzen algorithm, requires that the network satisfies the constraint that no continuous variables have discrete children [13]. As one would expect, this restriction places quite a burden on the generalization claim of the HBNs.

We believe that Fuzzy Set theory offers a comprehensive structure to introduce the ambiguity dimension of uncertainty to the existing framework of Hybrid Bayesian Networks and within this context, we are currently researching the development of a complete formalism that combines Fuzzy Sets and Bayesian Networks for reasoning about complex problems such as modeling the safety risk in UAS applications. Our proposed framework builds on the Lauritzen algorithm to generate a hybrid exact inferencing algorithm for general HBNs [14].

Conceptually, regardless of the resulting topological structure for the model of the complex system in question, the crisp-continuous variables in the hybrid Bayesian network are converted into their Fuzzy-discrete counterparts. This conceptual transformation of the model domain is depicted in Figure 6.

Consequently, the resulting Fuzzy-Bayesian network is propagated using the proposed probabilistic inferencing algorithm.

Notwithstanding particular emphasis on system safety analysis of UAS operations, the proposed hybrid methodology not only provides a tool set for realistic causal modeling of complex systems but also offers a calculus for quantifying complex 


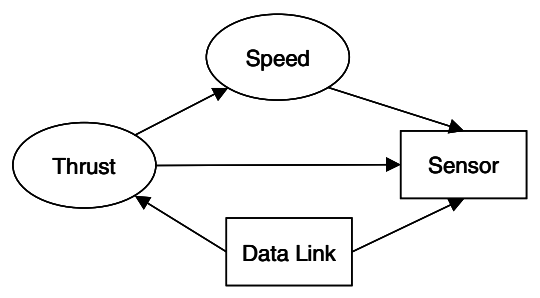

Hybrid Bayesian Network

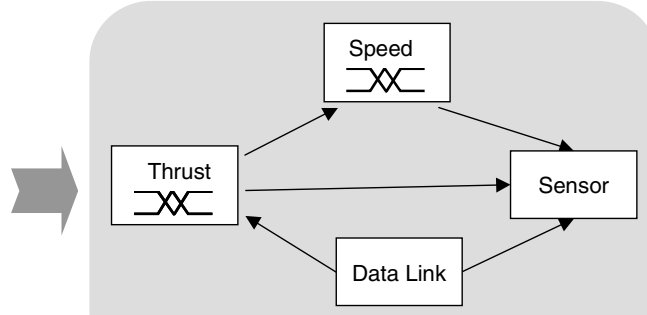

Fuzzy Bayesian Network

Fig. 4. The crisp domain of a HBN is transferred to the Fuzzy domain of the proposed FBN.

uncertainty in real-world applications. We are currently researching the development of a formalism that combines Fuzzy Sets and Bayesian Networks for reasoning about complex problems such as modeling the safety risk in UAS applications.

\section{Conclusions}

This paper outlines a conceptual framework for a regulatory-based system safety analysis of UAS integration into the NAS, with a particular emphasis on human factors while defining the building blocks of the proposed hazard and causal factor taxonomy. Within this context, four research components are proposed: HCAS taxonomy, regulatory-based causal-factor identification, database development, and uncertainty modeling of hybrid complex systems.

HCAS provides a systems-level hazard taxonomy developed for unmanned aircraft. It presents a structured framework to identify and classify or categorize both system and sub-system hazard sources for UAS operations. However hazard sources are not causal factors. In order to determine a taxonomy of UAS causal factors, we employ deductive reasoning and based our analysis on the current FAA regulations for commercial civil aviation. This taxonomy of causal factors will, in turn, constitute the seed for a database to facilitate safety analysis of immerging UAS operations. Finally, the concept of a hybrid fuzzy-Bayesian approach is outlined that is being developed to handle both discrete and continuous variables when uncertainty and vagueness may co-exist in the safety risk analysis.

Future research involves further development of the UAS causal factor taxonomy with the help of subject matter experts, detailed construction of the database using commercially available text mining tools, and more mathematical development of the hybrid methodology and applications to the unmanned aircraft contextual domain.

Acknowledgement. This research is supported by Federal Aviation Administration grant number 06-G-008. The contents of this paper reflect the views of the authors who are solely responsible for the accuracy of the facts, analyses, conclusions, and recommendations represented herein, and do not necessarily reflect the official view 
or policy of the Federal Aviation Administration. The authors acknowledge the support and participation of Dr. Xiaogong Lee, Mr. Michael Allocco, Mr. Steve Swartz, Mr. Robert Anoll and the FJ Leonelli Group, Inc. to the Rutgers research.

\section{References}

1. Roland, H.E., Moriarty, B.: System Safety Engineering and Management, 2nd edn. John Wiley \& Sons, Inc., New York (1990)

2. Heinrich, H.W.: Industrial Accident Prevention. McGraw Hill, New York (1936)

3. Rosenberg, B.: Next-Gen Nav/Comm. Aerospace Engineering and Manufacturing, 26-29 (2008)

4. Detect, Sense, and Avoid Safety Metrics (RTCA, RTCA Special Committee (SC) 203 Working Group 3, RTCA, Inc., Washington, DC, April 5 (2007a)

5. Guidance Material and Considerations for Unmanned Aircraft Systems (RTCA, DO-304, RTCA Special Committee (SC) 203, RTCA, Inc., Washington, DC, March 22 (2007b)

6. Öztekin, A., Luxhøj, J.T., Allocco, M.: A General Framework for Risk-Based System Safety Analysis of the Introduction of Emergent Aeronautical Operations into the National Airspace System. In: Baltimore, M.D. (ed.) Proceedings of the 25th International System Safety Conference, Baltimore, MD, August 13-17 (2007)

7. Hayhurst, K.J., Maddalon, J.M., Miner, P.S., DeEalt, M.P., McCormick, G.F.: Unmanned Aircraft Hazards and Their Implications for Regulation. In: 25th Digital Avionics Systems Conference, vol. 12, pp. 5B1-1 - 5B1-12 (2006)

8. De Jong, H.H., Blom, H., Stroeve, S.H.: How to Identify Unimaginable Hazards? In: Proceedings of the 25th International System Safety Conference, Baltimore, MD, August 1317 (2007)

9. Leveson, N.G.: Safeware: System Safety and Computers. Addison-Wesley Publishing Company, New York (1995)

10. Öztekin, A., Luxhøj, J.T.: Hazard, Safety Risk, and Uncertainty Modeling of the Integration of Unmanned Aircraft Systems into the National Airspace. In: $26^{\text {th }}$ Congress of International Council of the Aeronautical Sciences, Anchorage, Alaska, September 14-29 (2008)

11. Ross, T.: Fuzzy logic with engineering applications. McGraw-Hill, New York (1995)

12. Zadeh, L.: Fuzzy sets. Inf. Control 8, 338-353 (1965)

13. Lauritzen, S.: Propagation of probabilities, means, and variances in mixed graphical association models. JASA 87(420), 1089-1108 (1992)

14. Öztekin, A.: A Generalized Hybrid Fuzzy-Bayesian Methodology for Modeling Complex Uncertainty, Ph.D. Proposal, Department of Industrial and Systems Engineering, Rutgers University (August 2008) 